\title{
Prediction of physico-chemical properties for polycyclic aromatic hydrocarbons based on electronic characteristics of molecules
}

\author{
(C) Mikhail Yu. Dolomatov, ${ }^{1,2} *$ Natalia H. Paymurzina, ${ }^{1}$ and Ella A. Kovaleva ${ }^{1+}$ \\ ${ }^{1}$ Institute of Physics and Technology of Bashkir State University. Zaki Validi St., 32. \\ Ufa, 450074. Bashkortostan Republic. Russia. \\ ${ }^{2}$ Ufa State Petroleum Technological University. 1, Kosmonavtov St.,. Ufa, 450062. Bashkortostan Republic. \\ Russia.E-mail:mdolomatov@bk.ru,paimurzina@inbox.ru,kovaleva-ugntu@yandex.ru
}

\begin{abstract}
*Supervising author; ${ }^{+}$Corresponding author
Keywords: PAH, QSPR, integral spectroscopic descriptors, ionization potential, electron affinity, boiling point, saturation vapor pressure.
\end{abstract}

\begin{abstract}
QSPR models have been developed to predict of polycyclic aromatic hydrocarbons (PAHs) based on quantum chemical and integral spectroscopic descriptors. The first ionization potentials calculated from the energies of the highest occupied molecular orbital (HOMO), relative autocorrelation empirical parameters and the total number of electrons of non-ionized molecules were used as quantum chemical descriptors. Ionization potentials, electron affinities, boiling points, molecular masses, saturation vapor pressure of PAHs were studied as physical-chemical properties. Ionization potentials and electron affinities (IPs and EAs) are calculated by the use of density functional theory (DFT). Relative empirical autocorrelation parameters $\mu$ were calculated from the spectra of PAH molecules experimentally obtained and taken from the databases. The predictive power of resulting model is demonstrated by testing it on unseen data that were not used during model generation. Coefficients of determination for all dependencies considered in this paper are not less than 0.95. To assess the reliability for coefficients of determination, their mean errors were calculated. In this paper, we compared theoretical (experimental) and model-calculated values. Relative errors were estimated: for the electron donating ability of PAHs is $1.11 \%$ (for IP) and $0.86 \%$ (for EA); at about $3.1 \%$ for boiling points; not more than $0.51 \%$ for molar mass, for saturated vapor pressure, the error is more significant, which is apparently due to the difficulties of determining this value. The obtained models make it possible to estimate physical and chemical properties with sufficient accuracy for practical applications. The research results can be practically used in petrochemistry, carbon chemistry, organic chemistry, for prediction of physical and chemical properties $\mathrm{PAH}$ molecules.
\end{abstract}

\section{References}

[1] S.V. Dezortsev, L.I. Manzullina, K.I. Netsvetaeva, A.M. Petrov. Connection of physical- chemical properties with tye first ionization potential in homologous series benzene-penthacene. Bashkir chemical journal. 2014. Vol.21. No.4. P.33-39.

[2] M.Yu. Dolomatov, N.H. Paymurzina, and E.A. Kovaleva. Evaluation of donor-acceptor properties of polycyclic hydrocarbon molecules by the integral autocorrelation characteristics of the optical spectra. Butlerov Communications. 2018. Vol.53. No.2. P.28-37. DOI: 10.37952/ROI-jbc-01/18-53-2-28

[3] M.Yu. Dolomatov, K.F. Latypov, and E.A. Kovaleva. Prediction of vertical ionization potentials for organic compounds by integral characteristics of optical spectra and the number of protons in molecules. Butlerov Communications. 2019. Vol.58. No.6. P.62-72. DOI: 10.37952/ROI-jbc-01/19-58-6-62

[4] Patent Russia, no. 2621470, 06.06.2017. Method for determining the ionization potential of polycyclic aromatic hydrocarbon molecules. M.Y. Dolomatov, N.H. Paymurzina, E.A. Kovaleva.

[5] K.K. Tsymbaliuk, Iu.M. Den'ga, V.P. Antonovich. Determination of polycyclic aromatic hydrocarbons (PAHs) in the environment (Review). Methods and objects of chemical analysis. 2013. Vol.8. No.2. P.50-62. (russian)

[6] Mackay D.; Shiu, Wan Ying; Ma, Kuo-Ching; Lee, Sum Chi. Handbook of Physical-Chemical Properties and Environmental Fate for Organic Chemicals. Vol. 4. Nitrogen and Sulfur Containing Compounds and Pesticides. Second Edition - CRC Press- Taylor \& Francis Group. 2006. P.3195-4182

[7] NIST WebBook Chemistry http://webbook.nist.gov/chemistry

[8] Dortmund Data Bank http://www.ddbst.de/new/DDB/frame PCP.htm 